\title{
Tilt Sensor Based on Intermodal Photonic Crystal Fiber Interferometer
}

\author{
Xiaotong ZHANG, Kai NI, Chunliu ZHAO*, Manping YE, and Yongxing JIN \\ Institute of Optoelectronic Technology, China Jiliang University, Hangzhou, 310018, China \\ *Corresponding author: Chunliu ZHAO_E-mail: zhchunliu@hotmail.com
}

\begin{abstract}
A tilt sensor based on an intermodal photonic crystal fiber (PCF) interferometer is demonstrated. The sensor consists of a tubular filled with $\mathrm{NaCl}$ aqueous solutions and an intermodal PCF interferometer, which is formed by using a short PCF with two single-mode fibers (SMFs) spliced at both ends, and the air-holes in the splice regions are fully collapsed. The intermodal PCF interferometer is fixed in a rigid glass tubular with a slant orientation, and a half of the PCF is immersed in the $\mathrm{NaCl}$ aqueous solutions, while the other half is exposed in air. When tilting the tubular, the length of the PCF immersed changes so that the transmission spectrum moves. Therefore, by monitoring the wavelength shift, the tilt angle can be achieved. In the experiment, a $0.8-\mathrm{cm}$-length intermodal PCF interferometer was adopted. The sensitivity of the proposed sensor was obtained from $-1.5461 \mathrm{~nm} /{ }^{\circ}$ to $-30.1244 \mathrm{~nm} /{ }^{\circ}$ when measuring from $-35.1^{\circ}$ to $37.05^{\circ}$.
\end{abstract}

Keywords: Tilt sensors, photonic crystal fiber, interferometer, intermodal

Citation: Xiaotong ZHANG, Kai NI, Chunliu ZHAO, Manping YE, and Yongxing JIN, "Tilt Sensor Based on Intermodal Photonic Crystal Fiber Interferometer," Photonic Sensors, 2014, 4(3): 209-214.

\section{Introduction}

With the development of the measurement means, the measurement of the tilt angle has been needed in many fields, such as the level determination for mechanical guides, landing monitoring for the aircraft, and safety monitoring for petrochemical industry and electric power engineering. Most conventional tilt sensors are of an electronic type, transforming the inclination into electronic signals through a magnetic effect [1-4] or a capacitive effect [5]. Comparing with those tilt sensors above, optical fiber tilt sensors [6-13] have important advantages of electrically passive operation and immunity to electromagnetic interference, and most of the sensors are based on fiber Bragg gratings (FBGs). The principle of these sensors is that the FBGs' reflective wavelength will shift with applied strains through transitive installations when working. In the great majority of the tilt sensors based on the FBGs, the top ends of the FBGs are fixed on a frame while the bottom ends need to be bonded with a heavy mass [5-10]. These require the configuration of FBG-based tilt sensors to be more complex and delicate.

In this paper, we propose a tilt sensor based on an intermodal photonic crystal fiber (PCF) interferometer, which is easy for fabrications and with a compact and flexible construction. The intermodal PCF interferometer, as the sensor head, is formed by using a short PCF with two single-mode fibers (SMFs) spliced at both ends, and the air-holes in the splice regions are fully collapsed

\footnotetext{
Received: 5 March 2014 / Revised version: 11 May 2014

(C) The Author(s) 2014. This article is published with open access at Springerlink.com

DOI: $10.1007 / \mathrm{s} 13320-014-0186-\mathrm{z}$

Article type: Review
} 
by a fusion splicer. Then, the intermodal PCF interferometer is fixed in a rigid glass tubular with a slant orientation, and half of the PCF is in the $\mathrm{NaCl}$ aqueous solutions. When the sensor tilts, the length of the PCF immersed in the solutions changes leading to the dip wavelength of the PCF interference spectrum changes as well. So by monitoring the dip wavelength shift, we can obtain the tested tilt angle. Furthermore, the PCFs are highly insensitive to temperature, and the PCF used has a solid fiber core surrounded by periodical arrays of air holes in the fiber cladding. The tilt sensor has an advantage of a relatively higher thermal stability comparing with some sensors based on common fibers [14]. And experimental results show that the proposed tilt sensor works well when the tilt angles measured vary from $-35.1^{\circ}$ to $37.05^{\circ}$, and the sensitivity of the sensor is from -1.5461 $\mathrm{nm} /{ }^{\circ}$ to $-30.1244 \mathrm{~nm} /{ }^{\circ}$. And the tilt sensor proposed has good the temperature stability with the temperature varying.

\section{Setup of the tilt sensor}

Figure 1(a) shows the proposed sensor based on the intermodal PCF interferometer, in which the intermodal PCF interferometer passes through the glass tubular with a slant orientation and intersects with the central axis of the tubular. And the PCF's pigtails are glued on the wall of the tube to fix and keep it straight. The angle between the axis of the intermodal PCF interferometer and the central axis of the tubular is about $48^{\circ}$. The rigid glass tubular whose ends sealed is filled with $\mathrm{NaCl}$ aqueous solutions of which the refractive index is 1.38. Meanwhile, the liquid surface reaches to the place, where almost a half of the PCF is immersed into the liquid, and the other part is exposed to air. In order to eliminate the liquid surface bending and get a compact dimension, a glass tubular with $4.5 \mathrm{~cm}$ height and $3 \mathrm{~cm}$ diameter is chosen. The whole tilt sensor is placed on an adjustable platform. A superluminescent light-emitting diode (SLED) light source, whose wavelength range is from $1350 \mathrm{~nm}$ to $1680 \mathrm{~nm}$, is used as an input light source. The interference spectra of the proposed tilt sensor are measured by an optical spectrum analyzer (OSA, YOKOGAWA735301) with a spectral resolution of $0.02 \mathrm{~nm}$.

The cross section of the used PCF (NKT Photonics LMA-10, with four layers air-holes arrangement) is shown in Fig. 1(b). The diameter of the air hole is $3 \mu \mathrm{m}$ and the distance between two adjacent holes is $7 \mu \mathrm{m}$. And the cladding diameter is $62.5 \mu \mathrm{m}$. A short length of $0.8 \mathrm{~cm}$ of the PCF was utilized for the interferometer. A manual splicing between the PCF and the SMF, by adjusting the discharge current and duration, was used to fuse the glass of the PCF and distort the air-holes in the PCF cladding, as shown in Fig. 2. And a conventional fusion splicer (Sumitomo Type-39) was used for the fully collapsed splicing.
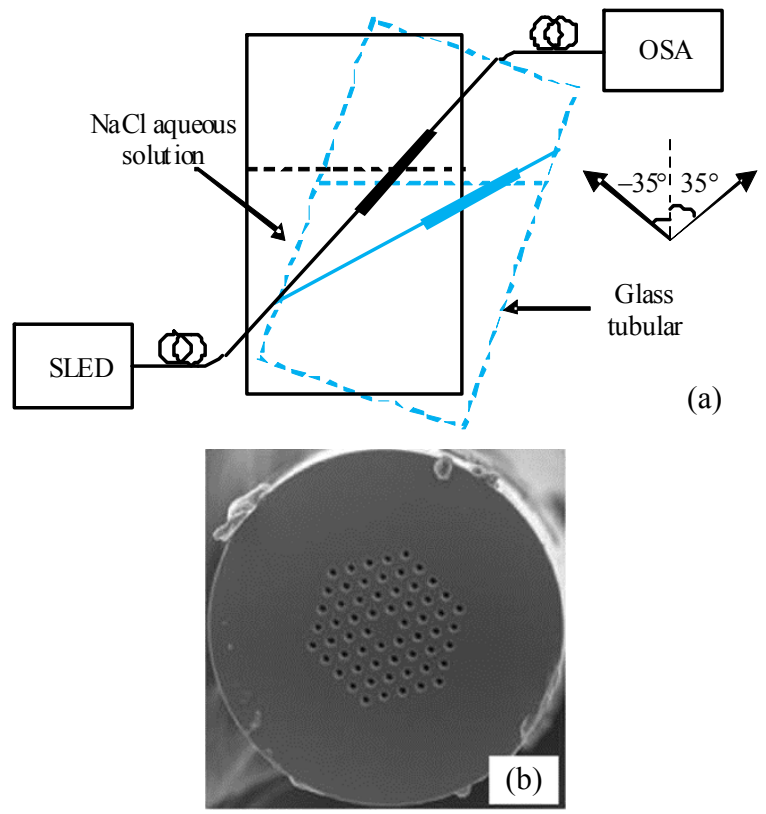

Fig. 1 Main setup of the tilt sensor: (a) schematic diagram of the proposed sensor and (b) SEM of the PCF.

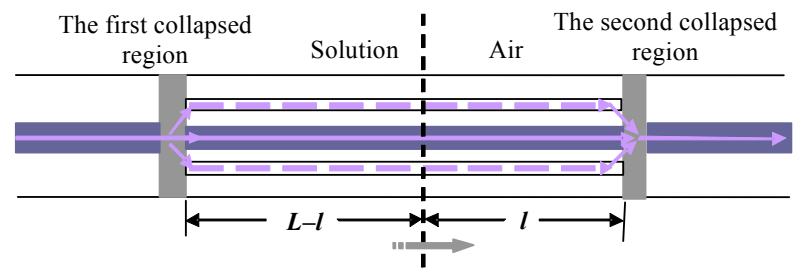

Fig. 2 Schematic diagram of the PCF interferometer. 


\section{Sensing principle}

Figure 2 shows the operating principle of the proposed tilt sensor based on the intermodal PCF interferometer. When the light from the SLED propagates through the first collapsed region of the PCF interferometer, different higher-order modes are excited. The fundamental mode and these higher-order modes propagate together through the PCF immersed into the solution at first, then through the part of PCF in air. At the second collapsed region of the PCF interferometer, the light with different modes recombine and interference due to different optical distances. As shown in Fig. 2, the optical distances of the fundamental mode and one higher-order mode are $n_{1} l+(L-l) n_{1}^{\prime}$ and $n_{m} l+(L-l) n_{m}^{\prime}$, respectively, where $n_{1}$ and $n_{m}$ are the effective indices of the fundamental mode and the certain higher-order mode when the PCF is exposed in air. Then, $n_{1}^{\prime}$ and $n_{m}^{\prime}$ are the effective indices of the fundamental mode and the certain higher mode in the solution. And $l$ is the length of the PCF in air, $L-l$ is the length of the PCF in the solution. The optical path difference $\Delta$ is obtained as

$$
\Delta=\left(n_{1}-n_{m}\right) l+\left(n_{1}^{\prime}-n_{m}^{\prime}\right)(L-l) .
$$

The transmission spectrum of the intermodal PCF interferometer is given as

$$
T=I_{1}+I_{2}+2 \sqrt{I_{1} I_{2}} \cos (2 \pi \Delta / \lambda)
$$

where $I_{1}$ and $I_{2}$ are the transmission intensities of two interfering modes, and $\lambda$ is the wavelength. And due to the relationship that $n_{1}$ is approximately equal to $n_{1}^{\prime}$, the wavelength dip of the transmission will exhibit and appear when

$$
\left(n_{1}-n_{m}^{\prime}\right) L+\left(n_{m}^{\prime}-n_{m}\right) l=(2 k+1) \lambda
$$

where $k$ is a natural number. When the sensor tilts, the length of the PCF exposed in air varies as well, and it eventually causes the dip wavelength moving. Therefore, the tested tilt angle can be obtained easily by measuring the wavelength dip.

In order to simplify the theory analysis, we transform the change in the liquid surface into a relative change between the liquid surface and tubular, as shown in Fig. 3.

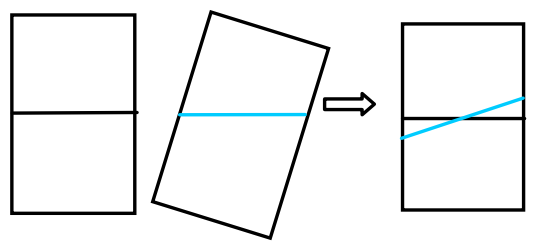

Fig. 3 Way used to analysis the liquid surface change.

Figure 4(a) shows different states of the tilt sensor when the tilt angle changes. The liquid surface will change when the sensor is tilting. Obviously, it is ease to prove that the liquid surfaces always pass the point $O$ which is the center of the liquid surface. As shown in Fig. 4(b), the line segment $O B$ stands for one liquid surface state with a tilt angle $\varphi$, and the point $B$ is the cross point of the liquid surface and the PCF. At this state, the length of the PCF in air equals to that of the line segment $A B$, and the PCF length in the solution is $B F$. The cross point of the liquid surface and the PCF will move along the fiber when the tilt angle is changed. According to the geometry, the relationship between $l$ and $\varphi$ is obtained as

$$
\frac{l-L / 2}{\sin \varphi}=\frac{O C}{\sin \left(48^{\circ}-\varphi\right)} .
$$

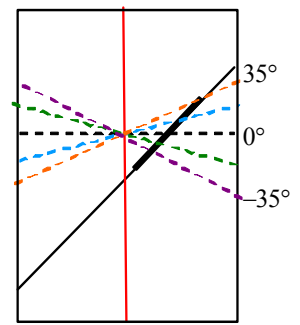

(a)

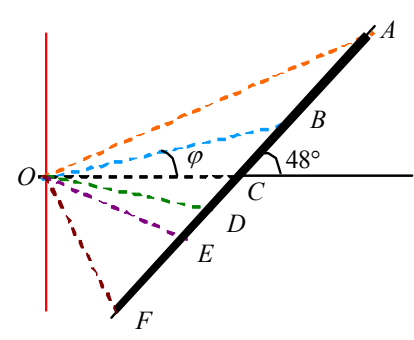

(b)
Fig. 4 Change in the liquid surface: (a) change in the liquid surface with different angles and (b) analysis of the relationship between the tilt angle and the length of the PCF in air.

Combining with (3), we can get the relationship between the tilt angle $\varphi$ and the wavelength $\lambda$ as below:

$$
\begin{aligned}
& \left(n_{m}-n_{m}^{\prime}\right) O C \frac{\sin \varphi}{\sin \left(48^{\circ}-\varphi\right)}+ \\
& \left(n_{1}-\frac{1}{2} n_{m}^{\prime}-\frac{1}{2} n_{m}\right) L=(2 k+1) \lambda
\end{aligned} .
$$

In our experiment, $O C$ is $1.1 \mathrm{~cm}$ long, and $L$ is 
$0.8 \mathrm{~cm}$ long. And when the wavelength is $1515 \mathrm{~nm}$, we get that $n_{1}$ is $1.442655, n_{m}$ is 1.439008 , and $n_{m}^{\prime}$ is 1.439011 through simulation with the software COMSOL. Then putting these values to (5), we can obtain that $y=1517.75-2.05 \sin x / \sin \left(48^{\circ}-x\right)$, where $y$ stands for the wavelength $\lambda$ and $x$ is the tilt angle $\varphi$. Therefore, by monitoring the dip wavelength of the intermodal PCF interferometer, we can obtain the measured tilt angles, which cause the change in the immersed length of the PCF.

\section{Experiment and results}

Figure 5 shows the whole transmission spectra of the tilt sensor based on the intermodal PCF interferometer when the tilt angles change from about $-35.1^{\circ}$ to $37.05^{\circ}$. There are two dips in the transmission spectra from the wavelength $1518 \mathrm{~nm}$ to $1565 \mathrm{~nm}$. At the tilt angle $0^{\circ}$, the two wavelength dips are at $1517.96 \mathrm{~nm}$ and $1566.59 \mathrm{~nm}$, respectively, and the extinction ratio reaches at about $15 \mathrm{~dB}$. The wavelength spacing between two adjacent dips is about $49 \mathrm{~nm}$. What's more, the transmission spectra have a blue-shift with the tilt angles increasing.

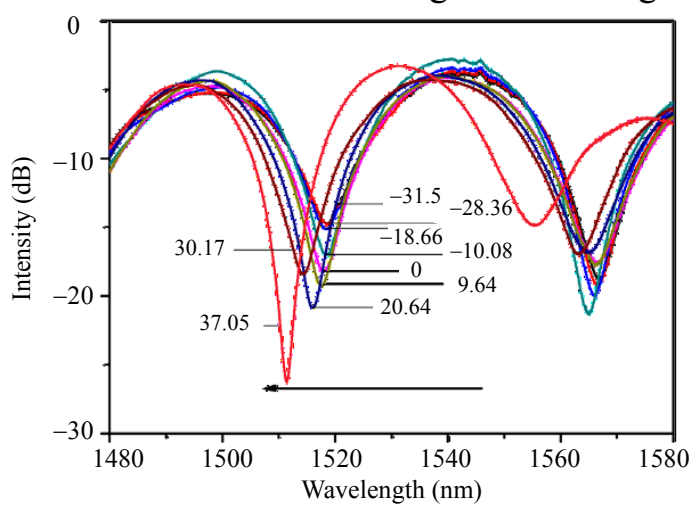

Fig. 5 Whole transmission spectra of the tilt sensor under different tilt angles (from $-35.1^{\circ}$ to $37.05^{\circ}$ ).

Figure 6 shows an enlargement of the wavelength dip of the transmission spectra at about $1518 \mathrm{~nm}$. As it is shown, from the wavelength 1490 $\mathrm{nm}$ to $1540 \mathrm{~nm}$, the dip of the transmission spectra has a blue-shift at different tilt angles in the range from $-35.1^{\circ}$ to $37.05^{\circ}$. Figure 7 shows the relationship between the dip wavelength and the tilt angle. The dip at $1518.80 \mathrm{~nm}$ of the PCF interference spectrum shifts to $1510.92 \mathrm{~nm}$ when the tilt angle varies from $-35.1^{\circ}$ to $37.05^{\circ}$. With the tilt angle increasing, the wavelength blue shifts larger and larger. When the tilt angle varies from $-35.1^{\circ}$ to $9.64^{\circ}$, the wavelength changing trend remains the same. However, when the tilt angle varies from $9.64^{\circ}$ to $37.05^{\circ}$, the trend changes rapidly. According to (5), the data's fitting curve is expressed as $y=1517.75-2.05 \sin x / \sin \left(48^{\circ}-x\right)$ with the fitting degree $R^{2}=0.984$. The results of the theory and experiment are consistent. And the sensitivity of the proposed tilt sensor is obtained as $y=$ $-2.05 \sin 48^{\circ} / \sin ^{2}\left(48^{\circ}-x\right)$, which is monotone increasing function within the range of degrees from about $-35^{\circ}$ to $37^{\circ}$. As shown in Fig. 8, the sensitivity curve of the tilt sensor falls gently when the tilt angle is less than $15^{\circ}$, yet falls precipitously when the tilt angle is more than $15^{\circ}$. For example, the sensitivity at the tilt angle $-35^{\circ}$ and $0^{\circ}$ are -1.5461 $\mathrm{nm} /{ }^{\circ}$ and $-2.7596 \mathrm{~nm} /{ }^{\circ}$, while it is $-30.1244 \mathrm{~nm} /{ }^{\circ}$ at the tilt angle of $37^{\circ}$. So, the tilt sensor has a large sensing range about $72^{\circ}$ and a high sensitivity especially when measuring a larger angle. The average precision of the tilt angle measurement is about $96.9 \%$.

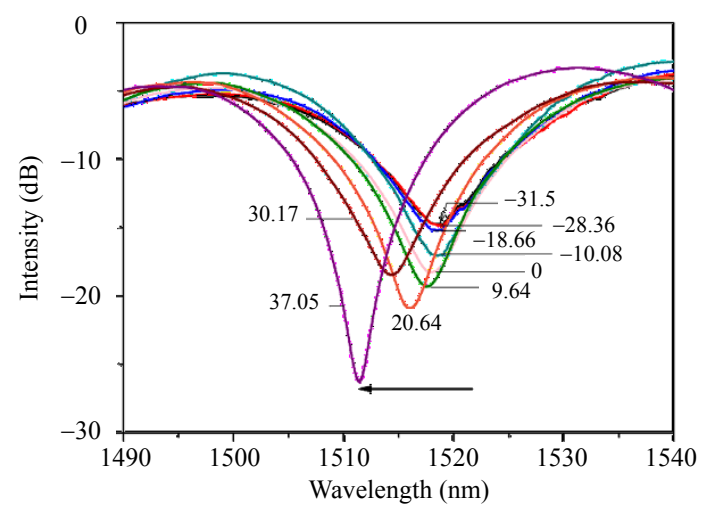

Fig. 6 Transmission spectra dip blue-shift under different tilt angles (from $-35.1^{\circ}$ to $37.05^{\circ}$ ).

The tilt angle is measured from $-35.1^{\circ}$ to $37.05^{\circ}$, which is just the angle formed by lines $O A$ and $O E$, as shown in Fig. 4(b). However, the largest tilt angle range can be measured is that formed by lines $O A$ and $O F$ in theory. This difference is caused by a lower sensitivity of a tilt angle less than $-35^{\circ}$, as 
shown in Fig. 8. To enlarge the tilt angle measurement range, the liquid surface should be lower properly. It can make the cross point between the liquid surface and the PCF move down along the PCF, so that the length of the PCF is fully utilized and able to measure a lager tilt angle. However, this behavior will bring a relatively low sensitivity due to the length of $O C$ becomes shorter. What's more, using a longer PCF is also an available way to increase the measurement range. Moreover, choosing a higher refractive index of the solution can increase the sensitivity of the proposed tilt sensor as well [15].

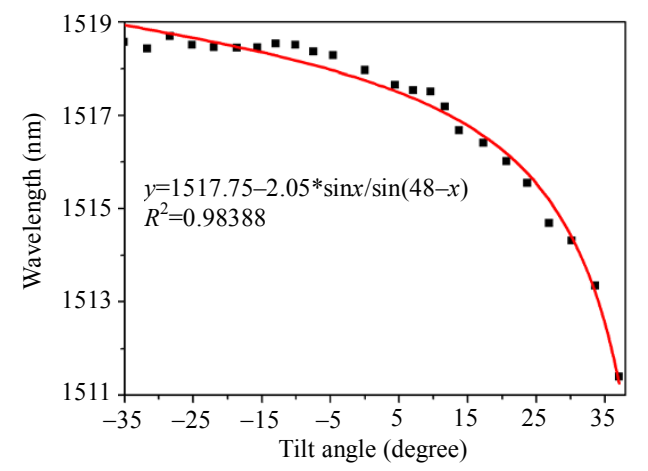

Fig. 7 Relationship of the dip wavelength with the tilt angle.

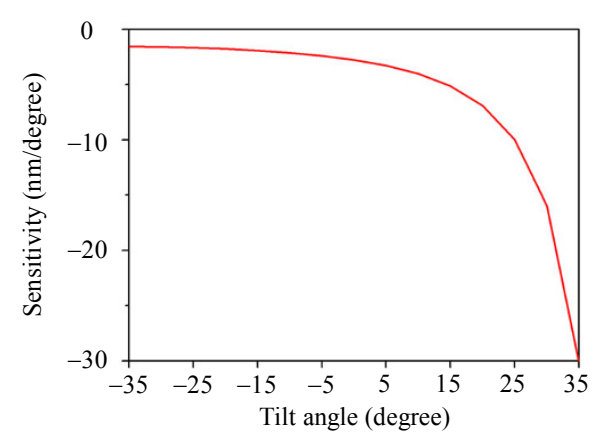

Fig. 8 Relationship of the sensitivity with the tilt angle.

In our experiment, the temperature characteristic of the intermodal PCF interferometer was measured by placing the whole intermodal PCF interferometer in a controlled temperature oven. And the temperature effect on the tilt sensor was measured in the temperature range from $20{ }^{\circ} \mathrm{C}$ to $80{ }^{\circ} \mathrm{C}$. As shown in Fig.9, the wavelength-temperature of the tilt sensor based on the intermodal PCF interferometer is about $5.8 \mathrm{pm} /{ }^{\circ} \mathrm{C}$. So the sensor has a good stability of temperature comparing with some sensors based on common fibers [14], In addition, the thermal index variation of the $\mathrm{NaCl}$ aqueous solution due to the influence of temperature in the range of $0.1{ }^{\circ} \mathrm{C}$ is about $1 \times 10^{-5}$ [16]. So the total temperature influence on the resonant wavelength drift of the intermodal PCF interferometer is small enough to be negligible. Generally, comparing with some sensors based on a common fiber, the sensor possesses a relatively higher stability of temperature.

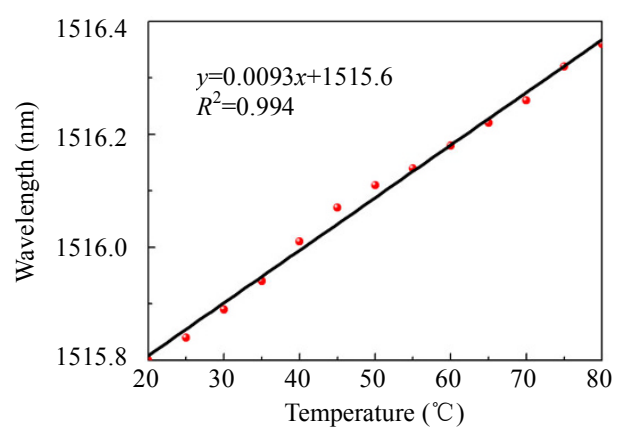

Fig. 9 Relationship of the dip wavelength with temperature.

\section{Conclusions}

A tilt sensor based on an intermodal PCF interferometer has been proposed and demonstrated experimentally. The intermodal PCF interferometer was formed by a short PCF fully collapsed spliced with SMFs. The sensitivity of the proposed tilt sensor in a large measurement range up to $72^{\circ}$ (from $-35.1^{\circ}$ to $37.05^{\circ}$ ) was achieved from $-1.5461 \mathrm{~nm} /{ }^{\circ}$ to $-30.1244 \mathrm{~nm} /{ }^{\circ}$ in the laboratory conditions. In addition, the temperature sensitivity of the proposed tilt sensor is relatively low. Comparing with other optical tilt sensors, the tilt sensor based on an intermodal PCF interferometer is of compact dimension and temperature stability, therefore, it has potential applications in a mass of static and quasi-static measurements.

\section{Acknowledgment}

This work was supported by the National Natural Science Foundation of China under Grant No. 61108058, the National Basic Research Program of China (973 Program) under Grant No. 2010CB327804, the Zhejing Province International 
Science and Technoloty Cooperation Special Programme under Grant No. 2013C24018, the National Key Technology R\&D Program 2011BAF06B02 and the Science and Technology Commission of Shanghai Municipality of China under Grant No. 10595812300.

Open Access This article is distributed under the terms of the Creative Commons Attribution License which permits any use, distribution, and reproduction in any medium, provided the original author(s) and source are credited.

\section{References}

[1] C. H. Lin and S. M. Kuo, "Micro-impedance inclinometer with wide-angle measuring capability and no damping effect," Sensors and Actuators A: Physical, 2008, 143(1): 113-119.

[2] J. Pyle and P. Emerald, "Convection-based technology offers the lowest-cost accelerometers and tilt sensors," AutoTechnology, 2002, 2(6): 60-63.

[3] T. Uchimura1, I. Towhata1, and T. T. L. Anh, "Simple monitoring method for precaution of landslides watching tilting and water contents on slopes surface," Landslides, 2010, 7(3): 351-357.

[4] D. Benz, T. Botzelmann, H. Kück, and D. Warkentin, "On low cost inclination sensors made from selectively metalized polymer," Sensors and Actuators A: Physical, 2005, 123-124: 18-22.

[5] R. Olaru and D. D. Dragoi, "Inductive tilt sensor with magnets and magnetic fluid," Sensors and Actuators A: Physical, 2005, 120(2): 424-428.

[6] B. O. Guan, H. Y. Tam, and S. Y. Liu, "Temperature-independent fiber Bragg grating tilt sensor," IEEE Photonics Technology Letters, 2004, 16(1): 224-226.
[7] S. L. He, X. Y. Dong, K. Ni, Y. X. Jin, C. C. Chan, and P. Shum, "Temperature-insensitive 2D tilt sensor with three fiber Bragg gratings," Measurement Science and Technology, 2010, 21(2): 025203.

[8] K. Ni, X. Y. Dong, Y. X. Jin, and H. S. Xu, "Temperature-independent fiber Bragg grating tilt sensor," Microwave and Optical Technology Letters, 2010, 52(10): 2250-2252.

[9] H. Y. Au, S. K. Khijwania, H. Y. Fu, W. H. Chung, and H. Y. Tam, "Temperature-insensitive fiber Bragg gratings based tilt sensor with large dynamic range," Journal of Lightwave Technology, 2011, 29(11): 1714-1720.

[10] H. L. Bao, X. Y. Dong, L. Y. Shao, C. L. Zhao, C. C. Chan, and P. Shum, "Temperature-insensitive pendulum clinometer using two fiber Bragg gratings for 2D tilt angle measurement," IEEE Photonic Technology Letter, 2010, 22(12): 863-865.

[11] H. J. Chen, L. Wang, and W. F. Liu, "Temperature-insensitive fiber Bragg grating tilt sensor," Applied Optics, 2008, 47(4): 556-560.

[12] X. Y. Dong, L. M. Hu, L. Y. Shao, Y. P. Wang, and J. Zheng, "Temperature-insensitive 2D fiber Bragg grating tilt sensor," Microwave and Optical Technology Letters, 2013, 55: 344-346.

[13] J. S. Bajić, D. Z. Stupar, A. Joža, M. P. Slankamenac, M. Jelić, and M.B. Živanov, "A simple fibre optic inclination sensor based on the refraction of light," Physica Scripta, 2012, T149: 014024.

[14] X. Y. Dong, C. L. Zhan, and K. Hu, "Temperatureinsensitive tilt sensor with strain-chirped fiber Bragg gratings," IEEE Photonics Technology Letter, 2005, 17(11): 2394-2396.

[15] W. W. Qian, C. C. Chan, and C. L. Zhao. "Photonic crystal fiber refractive index sensor based on a fiber Bragg grating demodulation," Sensors and Actuators $B, 2012,166-167:$ 761-765.

[16] D. R. Lide, CRC handbook of chemistry and physics, 82nd ed. Boca Raton, Florida: CRC Press, 2001. 